\title{
Axial Pressure Drop Measurements during Pilot-Scale Testing of a Mott Crossflow Filter
}

\author{
Michael R. Poirier and Samuel D. Fink \\ Savannah River Technology Center \\ Westinghouse Savannah River Company \\ Aiken, SC \\ and \\ Ralph Haggard, Travis Deal, Carol Stork and Vincent Van Brunt \\ Filtration Research Engineering Demonstration \\ Chemical Engineering Department \\ University of South Carolina \\ Columbia, SC
}

January 27, 2003 
This document was prepared in conjunction with work accomplished under Contract No. DE-AC09-96SR18500 with the U. S. Department of Energy.

\section{DISCLAIMER}

This report was prepared as an account of work sponsored by an agency of the United States Government. Neither the United States Government nor any agency thereof, nor any of their employees, makes any warranty, express or implied, or assumes any legal liability or responsibility for the accuracy, completeness, or usefulness of any information, apparatus, product or process disclosed, or represents that its use would not infringe privately owned rights. Reference herein to any specific commercial product, process or service by trade name, trademark, manufacturer, or otherwise does not necessarily constitute or imply its endorsement, recommendation, or favoring by the United States Government or any agency thereof. The views and opinions of authors expressed herein do not necessarily state or reflect those of the United States Government or any agency thereof.

This report has been reproduced directly from the best available copy.

Available for sale to the public, in paper, from: U.S. Department of Commerce, National Technical Information Service, 5285 Port Royal Road, Springfield, VA 22161, phone: (800) 553-6847, fax: (703) 605-6900

email: orders@ntis.fedworld.gov

online ordering: http://www.ntis.gov/help/index.asp

Available electronically at http://www.osti.gov/bridge

Available for a processing fee to U.S. Department of Energy and its contractors, in paper, from: U.S. Department of Energy, Office of Scientific and Technical Information, P.O. Box 62, Oak Ridge, TN 37831-0062,

phone: (865)576-8401,

fax: (865)576-5728

email: $\underline{\text { reports@ adonis.osti.gov }}$ 


\section{SUMMARY}

The Department of Energy selected caustic side solvent extraction (CSSX) as the preferred cesium removal technology for Savannah River Site waste. As a pretreatment step for the CSSX flowsheet, personnel contact the incoming salt solution that contains entrained sludge with monosodium titanate (MST) to adsorb strontium and select actinides. They filter the resulting slurry to remove the sludge and MST. We conducted pilot-scale crossflow filter testing with simulated SRS high level waste to evaluate the impact of operating parameters on the crossflow filtration process. During the tests, we measured the axial pressure drop as a function of axial velocity, feed slurry [i.e., sludge plus MST (5.6 M sodium), sludge plus MST (6.4 M sodium), sludge only, and sludge plus manganese dioxide], and insoluble solids concentration.

The conclusions from this work follow.

- The axial pressure drop varies with velocity ${ }^{1.75}$, which agrees with theory.

- The axial pressure drop depends on the feed slurry, but the effect is less than observed for axial velocity. Sludge plus manganese oxide slurries produce the highest axial pressure drop and sludge plus MST slurries produce the lowest axial pressure drop.

- The effect of insoluble solids concentration is statistically insignificant. However, if the insoluble solids loading increased over the levels in these tests and the slurry became nonNewtonian, concentration may have a significant effect on axial pressure drop.

- The measured axial pressure drops are approximately $2 X$ the value predicted based on pipe flow models. This observation agrees with similar data provided by a crossflow filter manufacturer.

\section{INTRODUCTION}

The Department of Energy selected CSSX as the preferred cesium removal technology for Savannah River Site waste. As a pretreatment step for the CSSX flowsheet, personnel contact the incoming salt solution that contains entrained sludge with MST to adsorb strontium and select actinides. They filter the resulting slurry to remove the sludge and MST. The filtrate receives further treatment in the solvent extraction system. The baseline filtration technology uses a Mott crossflow filter.

We conducted pilot-scale crossflow filter testing with simulated SRS high level waste to evaluate the impact of operating parameters on the crossflow filtration process. ${ }^{1,2,3,4}$ The feed slurries for these tests included simulated sludge plus MST, simulated sludge only, and simulated sludge plus manganese oxide solids. The supernate for these tests consisted of 5.6-6.4 M sodium, average salt solution. During the tests, we measured the axial pressure drop as a function of axial velocity, feed slurry, and insoluble solids concentration. This report documents the axial pressure drop data.

\section{TESTING}

The testing occurred at the Filtration Research Engineering Demonstration (FRED) facility at the University of South Carolina shown in Figure 1. The FRED facility contains a filter element 
with seven Mott filter tubes. Each tube is made from sintered stainless steel, 0.75 inches OD, 0.625 inches ID, 10 feet long, and nominal 0.5 micron pore size.

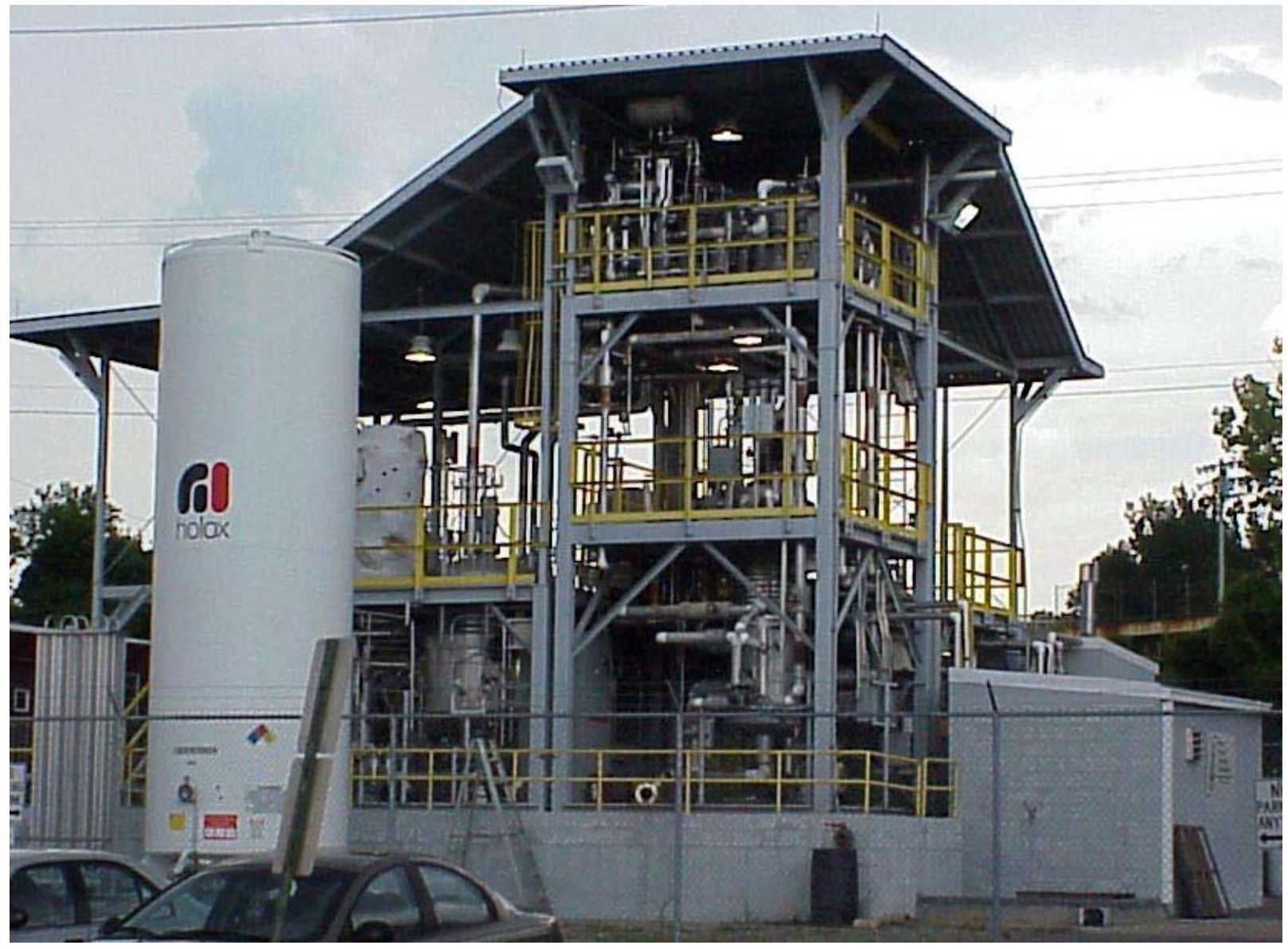

Figure 1. Filtration Research Engineering Demonstration

The simulated waste slurry contained nominally "average" Savannah River Site salt solution (5.6 -6.4 M sodium). Table 1 shows the feed slurries along with the insoluble solids loadings.

Personnel set the tank temperature to $35 \pm 3{ }^{\circ} \mathrm{C}$, and added feed solids to reach the target concentration. They circulated the feed solution through the system to mix it.

Details of the filter test protocol are described in the individual test reports. ${ }^{1-4}$ In some tests, personnel backpulsed the filter at the beginning of each test. The duration of the tests varied. These differences would affect the relationship between filter operating parameters and filter flux, but should have minimal impact on the relationship between filter axial velocity and axial pressure drop. 
Table 1. Feed Slurries and Insoluble Solids Loadings

\begin{tabular}{lcc} 
Feed Slurry & Na $(\mathrm{M})$ & Insoluble Solids (wt \%) \\
\hline Purex Sludge + MST & 6.4 & 0.05 \\
Purex Sludge + MST & 6.4 & 0.74 \\
Purex Sludge + MST & 6.4 & 0.05 \\
Purex Sludge + MST & 6.4 & 0.10 \\
Purex Sludge + MST & 6.4 & 0.20 \\
Purex Sludge + MST & 6.4 & 0.39 \\
Tank 40H/8F Sludge + MST & 5.6 & 0.033 \\
Tank 40H/8F Sludge + MST & 5.6 & 0.25 \\
Tank 40H/8F Sludge + MST & 5.6 & 1.1 \\
Tank 40H/8F Sludge + MST & 5.6 & 4.2 \\
Tank 8F Sludge & 5.6 & 0.044 \\
Tank 8F Sludge & 5.6 & 0.21 \\
Tank 8F Sludge & 5.6 & 0.88 \\
Tank 8F Sludge & 5.6 & 4.8 \\
Tank 40H Sludge + Manganese Oxide & 5.6 & 0.09 \\
Tank 40H Sludge + Manganese Oxide & 5.6 & 0.34 \\
Tank 40H Sludge + Manganese Oxide & 5.6 & 1.5 \\
Tank 40H Sludge + Manganese Oxide & 5.6 & 3.0
\end{tabular}

\section{RESULTS}

Figure 2 shows the axial pressure drop as a function of axial velocity in the tests with $6.4 \mathrm{M}$ sodium supernate, Purex sludge, and MST. (Attachment 1 contains the raw data.)

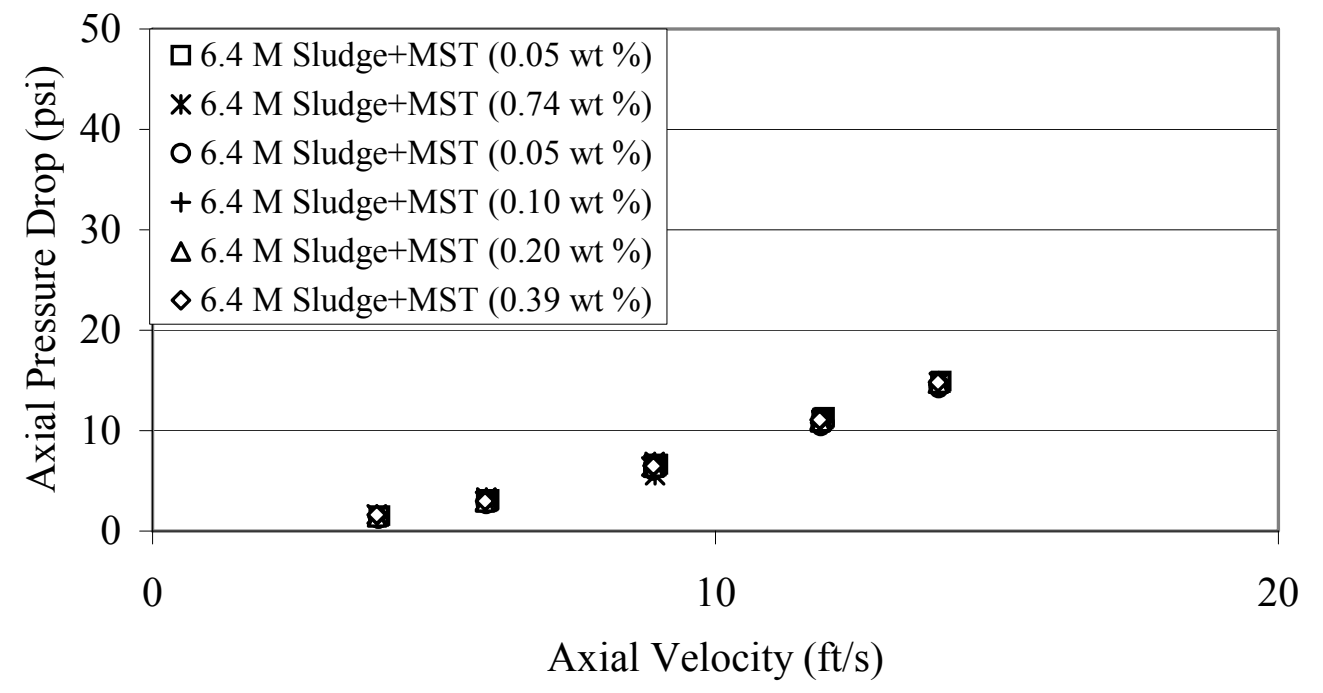

Figure 2. Axial Pressure Drop during Test with 6.4 M Sodium Supernate, Purex Sludge, and MST

Figure 3 shows the axial pressure drop as a function of axial velocity in the tests with $5.6 \mathrm{M}$ sodium supernate, Tank 40H sludge, Tank 8F sludge and MST. 


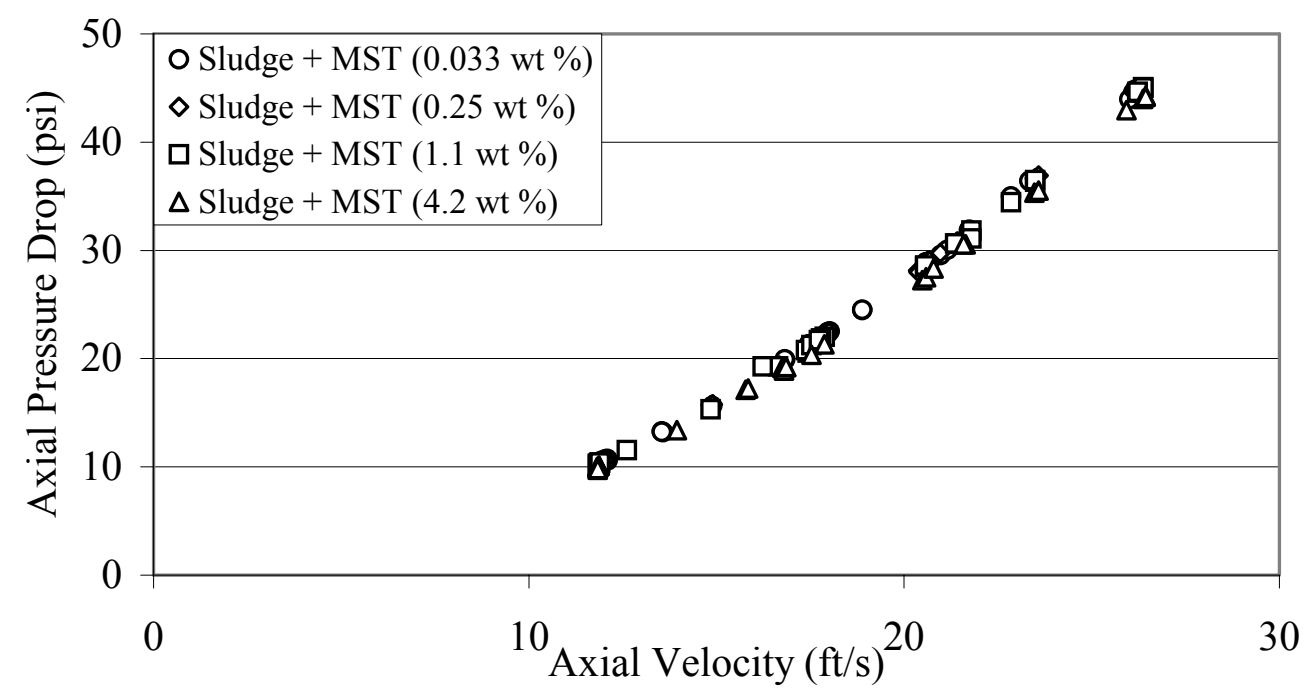

Figure 3. Axial Pressure Drop during Test with 5.6 M Sodium Supernate, Tank 40H Sludge, Tank 8F Sludge, and MST

Figure 4 shows the axial pressure drop as a function of axial velocity in the tests with $5.6 \mathrm{M}$ sodium supernate and Tank 8F sludge.

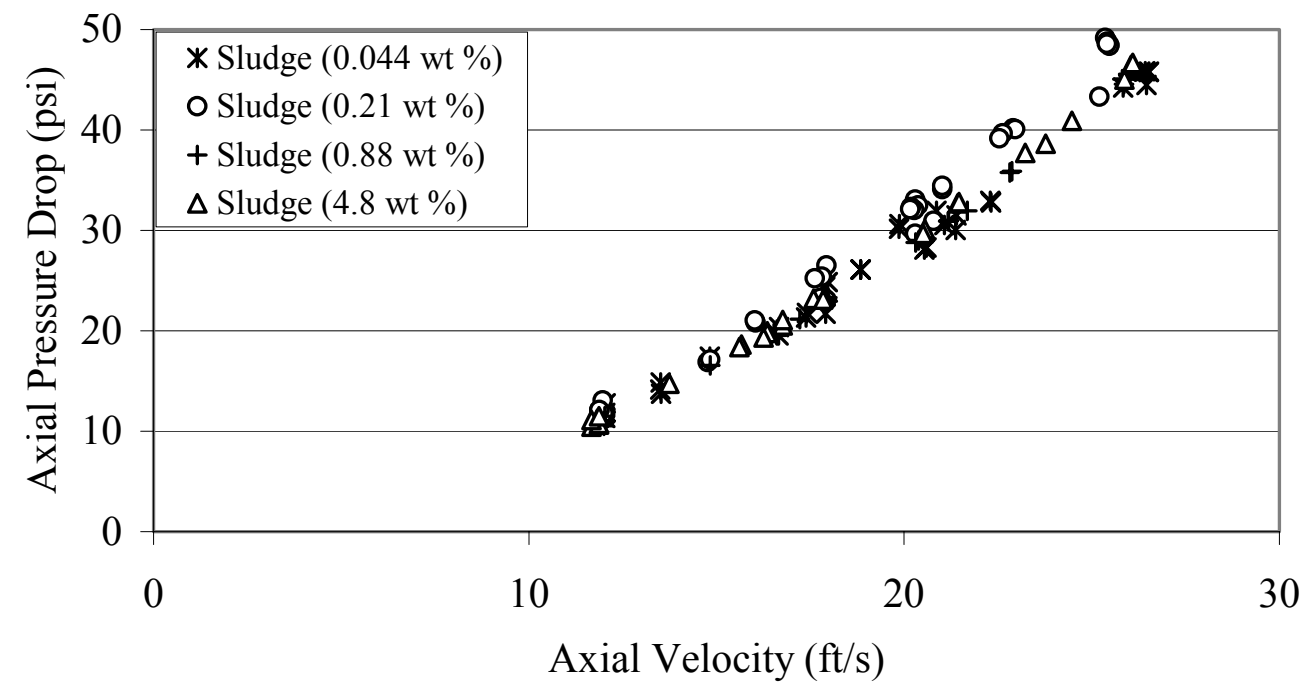

Figure 4. Axial Pressure Drop during Test with 5.6 M Sodium Supernate and Tank 8F Sludge

Figure 5 shows the axial pressure drop as a function of axial velocity in the tests with $5.6 \mathrm{M}$ sodium supernate, Tank $40 \mathrm{H}$ sludge, and manganese dioxide solids. 


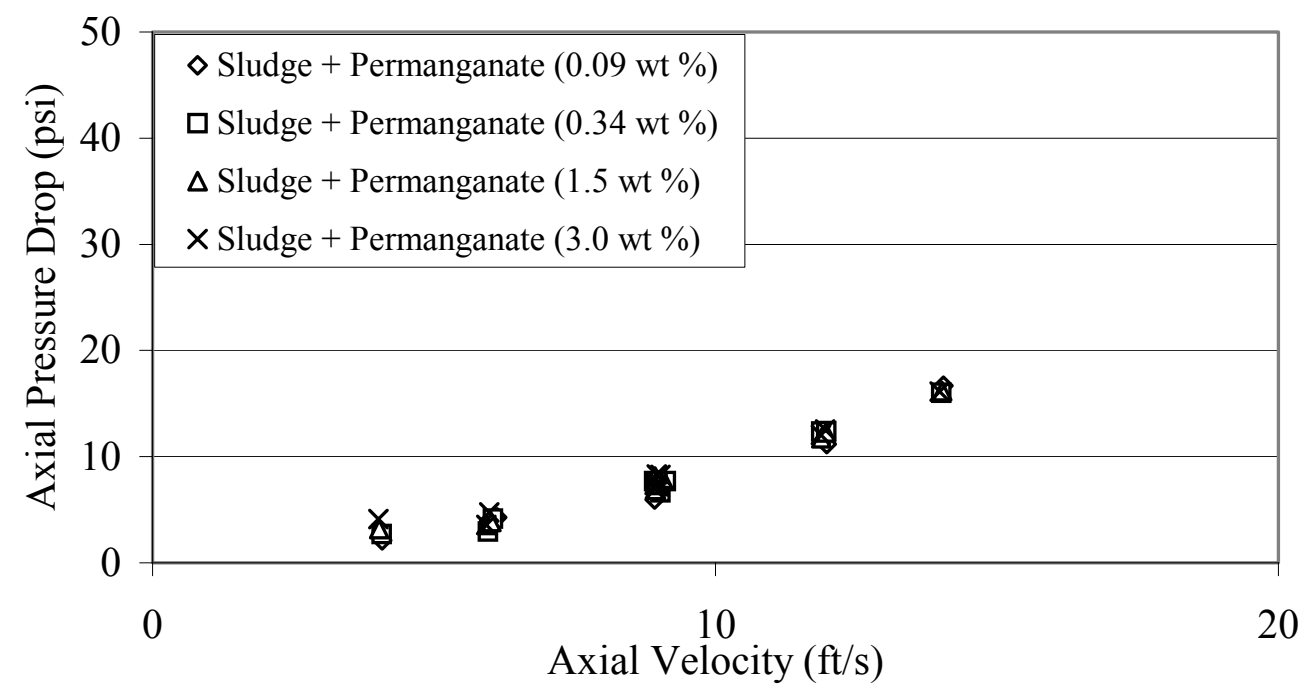

Figure 5. Axial Pressure Drop during Test with 5.6 M Sodium Supernate and Tank 40H Sludge, and Manganese Oxide Solids

Figure 6 shows the data from the four tests plotted together along with a theoretical prediction. One may calculate the axial velocity for turbulent pipe flow with equation [1]

$$
-\Delta P=\frac{0.158 v^{1.75} \rho^{0.75} L \mu^{0.25}}{D^{1.25}} \quad \text { for } \operatorname{Re}>10,000
$$

where $\mathrm{v}$ is axial velocity, $\rho$ is fluid density, $\mathrm{L}$ is tube length, $\mu$ is viscosity, $\mathrm{D}$ is tube diameter, and $R e$ is the Reynolds number. ${ }^{5}$ With the pilot-scale filter unit $(D=0.625$ in) and the SRS supernate $(\rho=1.26 \mathrm{~g} / \mathrm{mL}$ and $\mu=2.42 \mathrm{cp}$ ), an axial velocity of $4 \mathrm{ft} / \mathrm{s}$ produces a Reynolds number of 10,000 . Since the minimum axial velocity tested equaled $4 \mathrm{ft} / \mathrm{s}$, equation [1] applies.

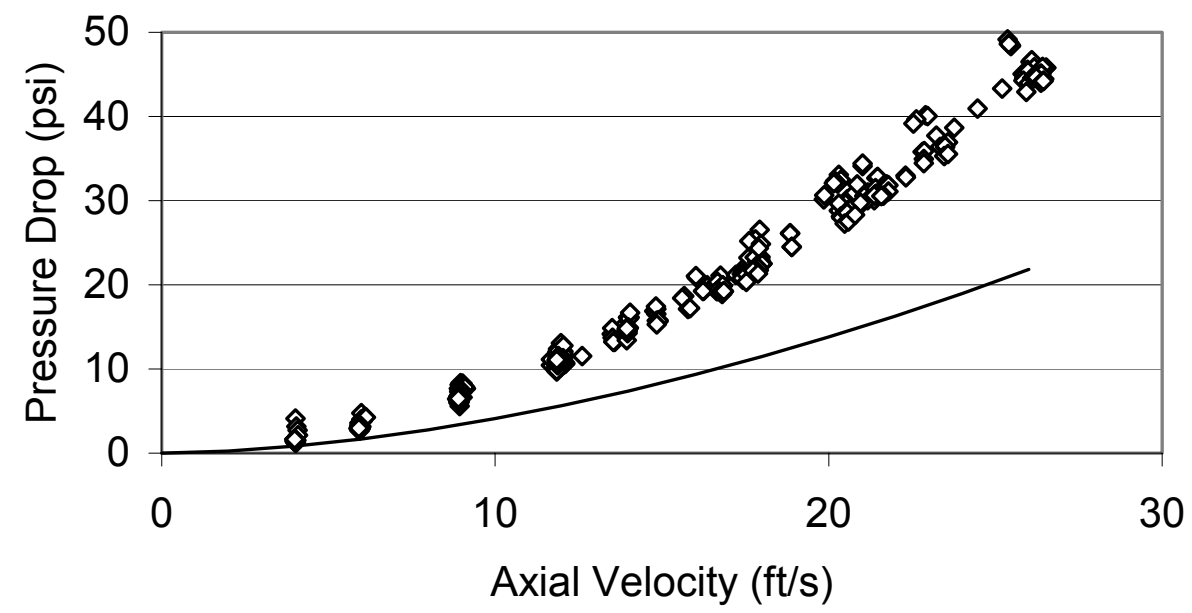

Figure 6. Axial Pressure Drop 
The pressure drops measured with the pilot-scale filter are approximately $2 \mathrm{X}$ the value predicted by equation [1]. This observation agrees with data in the User's Manual for SCT Membranes produced by Membralox ${ }^{\circledR}$, a manufacturer of crossflow filters. ${ }^{6}$

We conducted a statistical analysis of the data collected using the JMP ${ }^{\circledR}$ statistical software to determine which operating parameters influence the axial pressure drop. We regressed the data with a model described by equations [2] and [3]

$$
\begin{aligned}
& -\Delta P=f(\text { feed }) C v^{1.75} \\
& \ln [-\Delta P]=a_{1} \ln [f(f \text { feed })]+a_{2} \ln [C]+a_{3} \ln \left(v^{1.75}\right)
\end{aligned}
$$

where $\mathrm{f}$ is a function describing the effect of the variation in feed solution, $\mathrm{C}$ is the insoluble solids concentration, and $\mathrm{a}_{1}, \mathrm{a}_{2}$, and $\mathrm{a}_{3}$ are constants. Table 2 shows the results of the statistical analysis.

Table 2. Statistical Analysis of Axial Pressure Drop Data

$\begin{array}{llllll}\text { Source } & \text { \# parameters } & \frac{\text { Degrees freedom }}{3} & \underline{\text { Sum Squares }} & \underline{\text { F-ratio }} & \frac{\text { Prob. }>\mathrm{F}}{<0.0001} \\ \text { Feed } & 3 & 3 & 1.56 & 102 & \\ \operatorname{Ln}[\mathrm{C}] & 1 & 1 & 0.00052 & 0.10 & 0.75 \\ \operatorname{Ln}\left[\mathrm{v}^{1.75}\right] & 1 & 1 & 127 & 25,000 & 0.0000\end{array}$

The F-ratio is the ratio of the mean square from the model to the mean square from the error. From the F-ratio, one can calculate the probability that the variation observed is due to error rather than to the parameter being investigated. If the probability is less than 0.05 , the variation is due to the parameter and the effect of the parameter is statistically significant. The effect of feed slurry and axial velocity are statistically significant, with the axial velocity having the strongest effect.

Concentration does not have a significant effect on pressure drop over the range investigated. If one increased the insoluble solids concentration, and the slurry became non-Newtonian (i.e., Bingham Plastic), the concentration effect would likely become significant and the axial pressure drop would increase with increasing concentration.

Table 3 shows the estimates of the coefficients in equation [3]. The purpose of estimating the coefficients is not to develop an empirical correlation describing the axial pressure drop in the FRED facility, but rather to determine which feed slurries produce the largest axial pressure drop and to verify that the exponents describing the concentration and axial velocity effects are good approximations. The results show $\mathrm{v}^{1.75}$ describes the effect of axial velocity on axial pressure drop well and that the effect of concentration on axial pressure drop is very small. The data also show that sludge plus manganese oxide slurries will produce the largest pressure drop followed by sludge only slurries, sludge plus MST (@6.4 M sodium) slurries, and sludge plus MST (@) 5.6 M sodium) slurries. 
Table 3. Coefficients for Axial Pressure Drop Model

Term

Intercept

Feed(6.4 M Sludge + MST)

Feed (Sludge + MST)

Feed (Sludge $+\mathrm{MnO} 2)$

$\operatorname{Ln}[\mathrm{C}\}$

$\operatorname{Ln}\left[\mathrm{v}^{1.75}\right]$
Estimate

$-2.0$

$-0.056$

$-0.077$

0.13

0.00073

1.026

\section{CONCLUSIONS}

The conclusions from this work follow.

- The axial pressure drop varies with velocity to the 1.75 power, which agrees with theory.

- The axial pressure drop depends on the feed slurry, but the effect is less than observed for axial velocity. Sludge plus manganese oxide slurries produce the highest axial pressure drop and sludge plus MST slurries produce the lowest axial pressure drop.

- The effect of insoluble solids concentration is statistically insignificant. However, if the insoluble solids loading increased over the levels in these tests and the slurry became nonNewtonian, concentration may have a significant effect on axial pressure drop.

- The measured axial pressure drops are approximately $2 \mathrm{X}$ the value predicted based on pipe flow models. This observation agrees with similar data provided by a crossflow filter manufacturer.

\section{REFERENCES}

1. Ralph Haggard, Travis Deal, Carol Stork, and Vince Van Brunt, "Final Report on the Crossflow Filter Testing for the Salt Disposition Alternative", USC-FRED-PSP-RPT-090-010, Rev. 0, December 4, 1998.

2. M. R. Poirier, "FY2000 FRED Test Report,"WSRC-TR-2001-00035, Rev. 0, January 11, 2001 and included report "Final Report on the Crossflow Filter Optimization with 5.6 M Sodium Salt Solution” (V. Van Brunt, C. Stork, T. Deal, and R. Haggard, USC-FREDPSP-RPT-09-0-015, December 20, 2000).

3. V. Van Brunt, R. Haggard, T. Deal, C. Stork, M. R. Poirier, and S. D. Fink, "Cross-Flow Filtration of Simulated High-Level Waste Sludge (Tank 8F)”, WSRC-TR-2001-00195, April 20, 2001.

4. M. R. Poirier, S. D. Fink, R. Haggard, V. Van Brunt, R. Haggard, T. Deal, and C. Stork, "Comparison of Crossflow Filtration Performance for Manganese Oxide/Sludge Mixtures and Monosodium Titanate/Sludge Mixtures", WSRC-TR-2002-00194, Rev. 0, April 19, 2002.

5. C. O. Bennett and J. E. Myers, Momentum, Heat, and Mass Transfer, $3^{\text {rd }}$ Ed., McGrawHiil New York, 1982, p.168,198.

6. User’s Manual, SCT Membranes, Membralox®, March 1987. 
Attachment 1

Crossflow Filter Axial Pressure Drop Data

\begin{tabular}{|c|c|c|c|c|c|}
\hline Test & $\underline{\text { Feed }}$ & $\underline{\text { Temp }}$ & Conc (wt \%) & $\underline{\operatorname{Vel}(\mathrm{ft} / \mathrm{s})}$ & $\underline{\mathrm{DP}}$ (psi) \\
\hline 66 & manganese oxide & 35 & 3.04 & 8.93 & 6.94 \\
\hline 65 & manganese oxide & 35 & 3.04 & 5.93 & 3.59 \\
\hline 64 & manganese oxide & 35 & 3.04 & 13.98 & 16.13 \\
\hline 63 & manganese oxide & 35 & 3.04 & 9.02 & 8.29 \\
\hline 62 & manganese oxide & 35 & 3.04 & 5.98 & 4.76 \\
\hline 61 & manganese oxide & 35 & 3.04 & 8.94 & 7.28 \\
\hline 60 & manganese oxide & 35 & 3.04 & 11.87 & 12.05 \\
\hline 59 & manganese oxide & 35 & 3.04 & 8.96 & 8.33 \\
\hline 58 & manganese oxide & 35 & 3.04 & 4.01 & 4.11 \\
\hline 57 & manganese oxide & 35 & 3.04 & 11.95 & 12.56 \\
\hline 56 & manganese oxide & 35 & 3.04 & 8.96 & 7.45 \\
\hline 55 & manganese oxide & 35 & 1.52 & 9.01 & 7.23 \\
\hline 54 & manganese oxide & 35 & 1.52 & 5.94 & 3.56 \\
\hline 53 & manganese oxide & 35 & 1.52 & 14.03 & 16.16 \\
\hline 52 & manganese oxide & 35 & 1.52 & 9.07 & 7.91 \\
\hline 51 & manganese oxide & 35 & 1.52 & 6.02 & 3.83 \\
\hline 50 & manganese oxide & 35 & 1.52 & 8.94 & 6.86 \\
\hline 49 & manganese oxide & 35 & 1.52 & 11.88 & 11.74 \\
\hline 48 & manganese oxide & 35 & 1.52 & 8.93 & 8.11 \\
\hline 47 & manganese oxide & 35 & 1.52 & 4.03 & 3.16 \\
\hline 46 & manganese oxide & 35 & 1.52 & 11.96 & 12.29 \\
\hline 45 & manganese oxide & 35 & 1.52 & 8.92 & 7.35 \\
\hline 44 & manganese oxide & 35 & 0.34 & 9.02 & 6.64 \\
\hline 43 & manganese oxide & 35 & 0.34 & 5.96 & 2.96 \\
\hline 42 & manganese oxide & 35 & 0.34 & 14.01 & 16.00 \\
\hline 41 & manganese oxide & 35 & 0.34 & 9.12 & 7.68 \\
\hline 40 & manganese oxide & 35 & 0.34 & 6.05 & 4.16 \\
\hline 39 & manganese oxide & 35 & 0.34 & 8.96 & 7.49 \\
\hline 38 & manganese oxide & 35 & 0.34 & 11.88 & 12.36 \\
\hline 37 & manganese oxide & 35 & 0.34 & 8.91 & 7.68 \\
\hline 36 & manganese oxide & 35 & 0.34 & 4.07 & 2.71 \\
\hline 35 & manganese oxide & 35 & 0.34 & 11.97 & 12.42 \\
\hline 34 & manganese oxide & 35 & 0.34 & 8.97 & 7.43 \\
\hline 33 & manganese oxide & 35 & 0.07 & 9.07 & 7.94 \\
\hline 32 & manganese oxide & 35 & 0.07 & 5.99 & 4.02 \\
\hline 31 & manganese oxide & 35 & 0.07 & 14.05 & 16.69 \\
\hline 30 & manganese oxide & 35 & 0.07 & 9.10 & 7.84 \\
\hline 29 & manganese oxide & 35 & 0.07 & 6.12 & 4.27 \\
\hline 28 & manganese oxide & 35 & 0.07 & 9.00 & 7.41 \\
\hline 27 & manganese oxide & 35 & 0.07 & 11.91 & 12.09 \\
\hline 26 & manganese oxide & 35 & 0.07 & 8.93 & 7.33 \\
\hline 25 & manganese oxide & 35 & 0.07 & 4.08 & 2.12 \\
\hline 24 & manganese oxide & 35 & 0.07 & 11.98 & 11.20 \\
\hline 23 & manganese oxide & 35 & 0.07 & 8.92 & 5.98 \\
\hline 22 & water & 50 & 0 & 9.88 & 4.28 \\
\hline 21 & water & 50 & 0 & 6.72 & 1.11 \\
\hline 20 & water & 50 & 0 & 14.95 & 10.64 \\
\hline 19 & water & 50 & 0 & 10.15 & 4.50 \\
\hline
\end{tabular}




\begin{tabular}{|c|c|c|c|c|c|}
\hline Test & Feed & Temp & Conc (wt \%) & $\underline{\operatorname{Vel}(\mathrm{ft} / \mathrm{s})}$ & $\underline{\mathrm{DP}}(\mathrm{psi})$ \\
\hline 18 & $\overline{\text { water }}$ & 50 & 0 & 7.11 & 2.22 \\
\hline 17 & water & 50 & 0 & 9.85 & 4.97 \\
\hline 16 & water & 50 & 0 & 12.61 & 8.55 \\
\hline 15 & water & 50 & 0 & 9.54 & 5.27 \\
\hline 14 & water & 50 & 0 & 5.00 & 1.99 \\
\hline 13 & water & 50 & 0 & 12.95 & 9.83 \\
\hline 12 & water & 50 & 0 & 9.80 & 6.11 \\
\hline 11 & water & 30 & 0 & 9.79 & 6.44 \\
\hline 10 & water & 30 & 0 & 6.66 & 3.37 \\
\hline 9 & water & 30 & 0 & 14.88 & 13.28 \\
\hline 8 & water & 30 & 0 & 10.03 & 6.55 \\
\hline 7 & water & 30 & 0 & 7.04 & 3.43 \\
\hline 6 & water & 30 & 0 & 9.80 & 5.95 \\
\hline 5 & water & 30 & 0 & 12.66 & 9.31 \\
\hline 4 & water & 30 & 0 & 9.54 & 5.35 \\
\hline 3 & water & 30 & 0 & 5.04 & 1.41 \\
\hline 2 & water & 30 & 0 & 13.01 & 9.74 \\
\hline 1 & water & 30 & 0 & 10.87 & 6.17 \\
\hline 150 & sludge only & 35 & 4.8 & 15.67 & 18.65 \\
\hline 149 & sludge only & 35 & 4.8 & 15.61 & 18.40 \\
\hline 148 & sludge only & 35 & 4.8 & 16.36 & 19.94 \\
\hline 147 & sludge only & 35 & 4.8 & 24.47 & 40.92 \\
\hline 146 & sludge only & 35 & 4.8 & 16.36 & 19.80 \\
\hline 145 & sludge only & 35 & 4.8 & 11.83 & 11.25 \\
\hline 144 & sludge only & 35 & 4.8 & 11.81 & 10.90 \\
\hline 143 & sludge only & 35 & 4.8 & 11.67 & 10.46 \\
\hline 142 & sludge only & 35 & 4.8 & 11.87 & 10.68 \\
\hline 141 & sludge only & 35 & 4.8 & 11.87 & 10.76 \\
\hline 140 & sludge only & 35 & 4.8 & 16.25 & 19.32 \\
\hline 139 & sludge only & 35 & 4.8 & 13.75 & 14.71 \\
\hline 138 & sludge only & 35 & 4.8 & 11.67 & 11.12 \\
\hline 137 & sludge only & 35 & 4.8 & 17.59 & 23.21 \\
\hline 136 & sludge only & 35 & 4.8 & 26.05 & 46.49 \\
\hline 135 & sludge only & 35 & 4.8 & 21.43 & 32.68 \\
\hline 134 & sludge only & 35 & 4.8 & 16.76 & 20.55 \\
\hline 133 & sludge only & 35 & 4.8 & 11.87 & 11.48 \\
\hline 132 & sludge only & 35 & 4.8 & 16.76 & 21.09 \\
\hline 131 & sludge only & 35 & 4.8 & 21.46 & 32.84 \\
\hline 130 & sludge only & 35 & 4.8 & 26.09 & 46.66 \\
\hline 129 & sludge only & 35 & 4.8 & 23.23 & 37.73 \\
\hline 128 & sludge only & 35 & 4.8 & 20.55 & 30.14 \\
\hline 127 & sludge only & 35 & 4.8 & 17.84 & 23.12 \\
\hline 126 & sludge only & 35 & 4.8 & 20.51 & 29.65 \\
\hline 125 & sludge only & 35 & 4.8 & 23.77 & 38.65 \\
\hline 124 & sludge only & 35 & 4.8 & 25.87 & 45.04 \\
\hline 122 & sludge only & 35 & 0.88 & 17.95 & 23.32 \\
\hline 121 & sludge only & 35 & 0.88 & 17.96 & 22.93 \\
\hline 120 & sludge only & 35 & 0.88 & 17.94 & 23.17 \\
\hline 118 & sludge only & 35 & 0.88 & 12.03 & 11.03 \\
\hline 117 & sludge only & 35 & 0.88 & 12.00 & 10.54 \\
\hline
\end{tabular}




\begin{tabular}{|c|c|c|c|c|c|}
\hline Test & Feed & Temp & Conc (wt \%) & $\underline{\operatorname{Vel}(\mathrm{ft} / \mathrm{s})}$ & $\underline{\mathrm{DP}}(\mathrm{psi})$ \\
\hline 116 & sludge only & 35 & 0.88 & 21.68 & 31.95 \\
\hline 115 & sludge only & 35 & 0.88 & 17.90 & 22.59 \\
\hline 114 & sludge only & 35 & 0.88 & 12.03 & 11.04 \\
\hline 113 & sludge only & 35 & 0.88 & 12.00 & 10.78 \\
\hline 112 & sludge only & 35 & 0.88 & 17.22 & 21.17 \\
\hline 111 & sludge only & 35 & 0.88 & 11.99 & 10.95 \\
\hline 110 & sludge only & 35 & 0.88 & 25.81 & 45.06 \\
\hline 109 & sludge only & 35 & 0.88 & 16.61 & 20.12 \\
\hline 108 & sludge only & 35 & 0.88 & 12.02 & 11.50 \\
\hline 107 & sludge only & 35 & 0.88 & 14.83 & 16.54 \\
\hline 106 & sludge only & 35 & 0.88 & 16.53 & 19.78 \\
\hline 104 & sludge only & 35 & 0.88 & 25.97 & 45.55 \\
\hline 103 & sludge only & 35 & 0.88 & 26.16 & 45.82 \\
\hline 102 & sludge only & 35 & 0.88 & 25.86 & 44.57 \\
\hline 101 & sludge only & 35 & 0.88 & 21.18 & 30.96 \\
\hline 100 & sludge only & 35 & 0.88 & 16.61 & 19.62 \\
\hline 99 & sludge only & 35 & 0.88 & 11.87 & 10.46 \\
\hline 98 & sludge only & 35 & 0.88 & 16.61 & 19.54 \\
\hline 97 & sludge only & 35 & 0.88 & 21.17 & 30.93 \\
\hline 96 & sludge only & 35 & 0.88 & 25.89 & 45.07 \\
\hline 95 & sludge only & 35 & 0.88 & 22.82 & 35.77 \\
\hline 94 & sludge only & 35 & 0.88 & 20.39 & 29.02 \\
\hline 93 & sludge only & 35 & 0.88 & 17.85 & 22.71 \\
\hline 92 & sludge only & 35 & 0.88 & 20.31 & 28.81 \\
\hline 91 & sludge only & 35 & 0.88 & 22.87 & 35.88 \\
\hline 90 & sludge only & 35 & 0.88 & 25.98 & 45.40 \\
\hline 87 & sludge only & 35 & 0.21 & 17.93 & 23.06 \\
\hline 86 & sludge only & 35 & 0.21 & 11.90 & 11.15 \\
\hline 85 & sludge only & 35 & 0.21 & 11.99 & 11.44 \\
\hline 84 & sludge only & 35 & 0.21 & 12.03 & 11.62 \\
\hline 83 & sludge only & 35 & 0.21 & 25.20 & 43.31 \\
\hline 82 & sludge only & 35 & 0.21 & 11.90 & 11.42 \\
\hline 81 & sludge only & 35 & 0.21 & 11.98 & 11.69 \\
\hline 80 & sludge only & 35 & 0.21 & 20.30 & 29.66 \\
\hline 79 & sludge only & 35 & 0.21 & 20.78 & 30.94 \\
\hline 78 & sludge only & 35 & 0.21 & 14.77 & 16.90 \\
\hline 77 & sludge only & 35 & 0.21 & 14.83 & 17.13 \\
\hline 76 & sludge only & 35 & 0.21 & 12.04 & 12.08 \\
\hline 75 & sludge only & 35 & 0.21 & 17.78 & 23.26 \\
\hline 74 & sludge only & 35 & 0.21 & 11.99 & 11.77 \\
\hline 73 & sludge only & 35 & 0.21 & 11.88 & 12.08 \\
\hline 72 & sludge only & 35 & 0.21 & 11.97 & 13.06 \\
\hline 71 & sludge only & 35 & 0.21 & 20.30 & 33.08 \\
\hline 70 & sludge only & 35 & 0.21 & 17.93 & 26.52 \\
\hline 69 & sludge only & 35 & 0.21 & 25.36 & 49.17 \\
\hline 68 & sludge only & 35 & 0.21 & 22.90 & 40.17 \\
\hline 67 & sludge only & 35 & 0.21 & 20.37 & 32.50 \\
\hline 66 & sludge only & 35 & 0.21 & 17.81 & 25.39 \\
\hline 65 & sludge only & 35 & 0.21 & 20.27 & 32.04 \\
\hline 64 & sludge only & 35 & 0.21 & 22.96 & 40.07 \\
\hline 63 & sludge only & 35 & 0.21 & 25.47 & 48.46 \\
\hline
\end{tabular}




\begin{tabular}{|c|c|c|c|c|c|}
\hline Test & Feed & Temp & Conc (wt \%) & Vel (ft/s) & $\underline{\mathrm{DP}}(\mathrm{psi})$ \\
\hline 62 & sludge only & 35 & 0.21 & 25.47 & 48.38 \\
\hline 61 & sludge only & 35 & 0.21 & 21.02 & 34.12 \\
\hline 60 & sludge only & 35 & 0.21 & 16.04 & 20.84 \\
\hline 59 & sludge only & 35 & 0.21 & 11.88 & 12.13 \\
\hline 58 & sludge only & 35 & 0.21 & 16.02 & 21.04 \\
\hline 57 & sludge only & 35 & 0.21 & 21.02 & 34.43 \\
\hline 56 & sludge only & 35 & 0.21 & 25.41 & 48.78 \\
\hline 55 & sludge only & 35 & 0.21 & 22.63 & 39.66 \\
\hline 54 & sludge only & 35 & 0.21 & 20.19 & 32.33 \\
\hline 53 & sludge only & 35 & 0.21 & 17.62 & 25.21 \\
\hline 52 & sludge only & 35 & 0.21 & 20.16 & 32.10 \\
\hline 51 & sludge only & 35 & 0.21 & 22.54 & 39.18 \\
\hline 50 & sludge only & 35 & 0.21 & 25.40 & 48.60 \\
\hline 42 & sludge only & 35 & 0.044 & 19.86 & 30.15 \\
\hline 41 & sludge only & 35 & 0.044 & 19.87 & 30.63 \\
\hline 40 & sludge only & 35 & 0.044 & 14.82 & 17.44 \\
\hline 39 & sludge only & 35 & 0.044 & 17.97 & 24.85 \\
\hline 38 & sludge only & 35 & 0.044 & 17.90 & 24.38 \\
\hline 37 & sludge only & 35 & 0.044 & 20.86 & 31.93 \\
\hline 36 & sludge only & 35 & 0.044 & 18.84 & 26.12 \\
\hline 35 & sludge only & 35 & 0.044 & 18.84 & 26.08 \\
\hline 34 & sludge only & 35 & 0.044 & 13.50 & 14.15 \\
\hline 33 & sludge only & 35 & 0.044 & 12.04 & 11.81 \\
\hline 32 & sludge only & 35 & 0.044 & 13.51 & 14.87 \\
\hline 31 & sludge only & 35 & 0.044 & 12.05 & 12.76 \\
\hline 30 & sludge only & 35 & 0.044 & 17.41 & 21.77 \\
\hline 29 & sludge only & 35 & 0.044 & 25.84 & 44.25 \\
\hline 28 & sludge only & 35 & 0.044 & 21.07 & 30.54 \\
\hline 27 & sludge only & 35 & 0.044 & 12.03 & 11.31 \\
\hline 26 & sludge only & 35 & 0.044 & 13.52 & 13.73 \\
\hline 25 & sludge only & 35 & 0.044 & 17.39 & 21.31 \\
\hline 24 & sludge only & 35 & 0.044 & 26.53 & 45.81 \\
\hline 23 & sludge only & 35 & 0.044 & 21.37 & 30.04 \\
\hline 22 & sludge only & 35 & 0.044 & 16.65 & 19.56 \\
\hline 21 & sludge only & 35 & 0.044 & 11.90 & 11.52 \\
\hline 20 & sludge only & 35 & 0.044 & 16.67 & 20.36 \\
\hline 19 & sludge only & 35 & 0.044 & 21.40 & 31.50 \\
\hline 18 & sludge only & 35 & 0.044 & 26.51 & 45.78 \\
\hline 17 & sludge only & 35 & 0.044 & 22.30 & 32.96 \\
\hline 16 & sludge only & 35 & 0.044 & 20.54 & 28.09 \\
\hline 15 & sludge only & 35 & 0.044 & 17.91 & 21.71 \\
\hline 14 & sludge only & 35 & 0.044 & 20.60 & 28.31 \\
\hline 13 & sludge only & 35 & 0.044 & 22.32 & 32.76 \\
\hline 12 & sludge only & 35 & 0.044 & 26.46 & 44.48 \\
\hline 11 & sludge only & 35 & 0.044 & 26.42 & 45.87 \\
\hline 30 & Sludge+MST & 35 & 0.033 & 26.10 & 44.53 \\
\hline 31 & Sludge+MST & 35 & 0.033 & 22.85 & 34.97 \\
\hline 32 & Sludge+MST & 35 & 0.033 & 20.59 & 28.91 \\
\hline 33 & Sludge+MST & 35 & 0.033 & 17.96 & 22.28 \\
\hline 34 & Sludge+MST & 35 & 0.033 & 20.69 & 28.99 \\
\hline
\end{tabular}




\begin{tabular}{|c|c|c|c|c|c|}
\hline Test & Feed & Temp & Conc (wt \%) & $\underline{\mathrm{Vel}}(\mathrm{ft} / \mathrm{s})$ & DP (psi) \\
\hline 35 & Sludge + MST & 35 & 0.033 & 23.34 & 36.40 \\
\hline 36 & Sludge+MST & 35 & 0.033 & 26.16 & 44.84 \\
\hline 37 & Sludge + MST & 35 & 0.033 & 21.74 & 31.92 \\
\hline 38 & Sludge+MST & 35 & 0.033 & 16.83 & 19.90 \\
\hline 39 & Sludge $+\mathrm{MST}$ & 35 & 0.033 & 11.93 & 10.54 \\
\hline 40 & Sludge+MST & 35 & 0.033 & 16.82 & 19.95 \\
\hline 41 & Sludge+MST & 35 & 0.033 & 21.73 & 31.82 \\
\hline 42 & Sludge+MST & 35 & 0.033 & 26.15 & 44.67 \\
\hline 43 & Sludge+MST & 35 & 0.033 & 16.67 & 19.24 \\
\hline 44 & Sludge+MST & 35 & 0.033 & 13.58 & 13.22 \\
\hline 45 & Sludge + MST & 35 & 0.033 & 14.89 & 15.64 \\
\hline 46 & Sludge+MST & 35 & 0.033 & 12.09 & 10.75 \\
\hline 47 & Sludge+MST & 35 & 0.033 & 13.55 & 13.29 \\
\hline 48 & Sludge+MST & 35 & 0.033 & 17.48 & 21.32 \\
\hline 49 & Sludge + MST & 35 & 0.033 & 18.89 & 24.49 \\
\hline 50 & Sludge + MST & 35 & 0.033 & 17.47 & 21.31 \\
\hline 51 & Sludge+MST & 35 & 0.033 & 18.89 & 24.52 \\
\hline 52 & Sludge $+\mathrm{MST}$ & 35 & 0.033 & 12.05 & 10.70 \\
\hline 53 & Sludge+MST & 35 & 0.033 & 13.55 & 13.24 \\
\hline 54 & Sludge + MST & 35 & 0.033 & 18.02 & 22.52 \\
\hline 55 & Sludge+MST & 35 & 0.033 & 17.97 & 22.43 \\
\hline 56 & Sludge+MST & 35 & 0.033 & 20.97 & 29.57 \\
\hline 57 & Sludge + MST & 35 & 0.033 & 26.00 & 43.97 \\
\hline 58 & Sludge + MST & 35 & 0.033 & 12.10 & 10.58 \\
\hline 59 & Sludge+MST & 35 & 0.033 & 21.16 & 30.07 \\
\hline 60 & Sludge+MST & 35 & 0.25 & 18.00 & 22.48 \\
\hline 61 & Sludge+MST & 35 & 0.25 & 26.27 & 45.02 \\
\hline 62 & Sludge + MST & 35 & 0.25 & 23.48 & 36.66 \\
\hline 63 & Sludge + MST & 35 & 0.25 & 20.63 & 29.01 \\
\hline 64 & Sludge $+\mathrm{MST}$ & 35 & 0.25 & 17.83 & 22.22 \\
\hline 65 & Sludge+MST & 35 & 0.25 & 20.67 & 29.08 \\
\hline 66 & Sludge + MST & 35 & 0.25 & 23.58 & 36.91 \\
\hline 67 & Sludge+MST & 35 & 0.25 & 26.25 & 44.93 \\
\hline 68 & Sludge+MST & 35 & 0.25 & 21.42 & 30.78 \\
\hline 69 & Sludge + MST & 35 & 0.25 & 16.68 & 19.36 \\
\hline 70 & Sludge + MST & 35 & 0.25 & 11.93 & 10.38 \\
\hline 71 & Sludge+MST & 35 & 0.25 & 12.09 & 10.62 \\
\hline 72 & Sludge+MST & 35 & 0.25 & 21.43 & 30.78 \\
\hline 73 & Sludge+MST & 35 & 0.25 & 26.27 & 44.79 \\
\hline 74 & Sludge+MST & 35 & 0.25 & 21.41 & 30.80 \\
\hline 75 & Sludge + MST & 35 & 0.25 & 20.40 & 28.10 \\
\hline 76 & Sludge + MST & 35 & 0.25 & 12.04 & 10.54 \\
\hline 77 & Sludge $+\mathrm{MST}$ & 35 & 0.25 & 11.97 & 10.24 \\
\hline 78 & Sludge+MST & 35 & 0.25 & 12.05 & 10.54 \\
\hline 79 & Sludge + MST & 35 & 0.25 & 17.80 & 21.87 \\
\hline 80 & Sludge+MST & 35 & 0.25 & 12.08 & 10.62 \\
\hline 81 & Sludge+MST & 35 & 0.25 & 14.90 & 15.77 \\
\hline 82 & Sludge + MST & 35 & 0.25 & 14.84 & 15.72 \\
\hline 83 & Sludge + MST & 35 & 0.25 & 20.96 & 29.74 \\
\hline 84 & Sludge+MST & 35 & 0.25 & 20.37 & 28.14 \\
\hline 85 & Sludge+MST & 35 & 0.25 & 12.03 & 10.62 \\
\hline
\end{tabular}




\begin{tabular}{|c|c|c|c|c|c|}
\hline$\underline{\text { Test }}$ & Feed & $\underline{\text { Temp }}$ & Conc (wt \%) & $\underline{V e l}(\mathrm{ft} / \mathrm{s})$ & $\underline{\mathrm{DP}}$ (psi) \\
\hline 86 & Sludge + MST & 35 & 0.25 & 11.98 & 10.48 \\
\hline 87 & Sludge + MST & 35 & 0.25 & 26.13 & 44.59 \\
\hline 88 & Sludge + MST & 35 & 0.25 & 12.07 & 10.71 \\
\hline 89 & Sludge + MST & 35 & 0.25 & 12.04 & 10.56 \\
\hline 90 & Sludge + MST & 35 & 0.25 & 11.97 & 10.34 \\
\hline 91 & Sludge + MST & 35 & 0.25 & 17.81 & 21.85 \\
\hline 92 & Sludge + MST & 35 & 0.25 & 14.89 & 15.74 \\
\hline 93 & Sludge+MST & 35 & 0.25 & 20.38 & 27.98 \\
\hline 94 & Sludge + MST & 35 & 1.1 & 26.30 & 44.64 \\
\hline 95 & Sludge + MST & 35 & 1.1 & 23.49 & 36.34 \\
\hline 96 & Sludge + MST & 35 & 1.1 & 20.56 & 28.49 \\
\hline 97 & Sludge + MST & 35 & 1.1 & 17.89 & 22.01 \\
\hline 98 & Sludge+MST & 35 & 1.1 & 20.56 & 28.58 \\
\hline 99 & Sludge+MST & 35 & 1.1 & 23.49 & 36.48 \\
\hline 100 & Sludge + MST & 35 & 1.1 & 26.37 & 45.09 \\
\hline 101 & Sludge + MST & 35 & 1.1 & 21.38 & 30.60 \\
\hline 102 & Sludge + MST & 35 & 1.1 & 16.64 & 19.28 \\
\hline 103 & Sludge + MST & 35 & 1.1 & 11.90 & 10.40 \\
\hline 104 & Sludge + MST & 35 & 1.1 & 16.23 & 19.27 \\
\hline 105 & Sludge+MST & 35 & 1.1 & 21.36 & 30.64 \\
\hline 106 & Sludge + MST & 35 & 1.1 & 26.38 & 45.03 \\
\hline 107 & Sludge + MST & 35 & 1.1 & 22.85 & 34.45 \\
\hline 108 & Sludge + MST & 35 & 1.1 & 12.61 & 11.54 \\
\hline 109 & Sludge + MST & 35 & 1.1 & 26.21 & 44.59 \\
\hline 110 & Sludge + MST & 35 & 1.1 & 11.85 & 10.10 \\
\hline 111 & Sludge + MST & 35 & 1.1 & 17.43 & 20.57 \\
\hline 112 & Sludge + MST & 35 & 1.1 & 14.84 & 15.32 \\
\hline 113 & Sludge + MST & 35 & 1.1 & 11.89 & 10.16 \\
\hline 114 & Sludge + MST & 35 & 1.1 & 17.39 & 20.80 \\
\hline 115 & Sludge + MST & 35 & 1.1 & 26.23 & 44.64 \\
\hline 116 & Sludge + MST & 35 & 1.1 & 11.85 & 10.22 \\
\hline 117 & Sludge + MST & 35 & 1.1 & 17.53 & 21.23 \\
\hline 118 & Sludge + MST & 35 & 1.1 & 11.85 & 10.28 \\
\hline 119 & Sludge + MST & 35 & 1.1 & 11.88 & 10.37 \\
\hline 120 & Sludge + MST & 35 & 1.1 & 17.79 & 21.86 \\
\hline 121 & Sludge + MST & 35 & 1.1 & 21.79 & 31.85 \\
\hline 122 & Sludge + MST & 35 & 1.1 & 11.85 & 10.29 \\
\hline 123 & Sludge + MST & 35 & 1.1 & 11.89 & 10.39 \\
\hline 124 & Sludge+MST & 35 & 1.1 & 17.73 & 21.69 \\
\hline 125 & Sludge + MST & 35 & 1.1 & 21.79 & 31.11 \\
\hline 126 & Sludge + MST & 35 & 4.2 & 26.37 & 43.99 \\
\hline 127 & Sludge + MST & 35 & 4.2 & 23.47 & 35.32 \\
\hline 128 & Sludge + MST & 35 & 4.2 & 20.48 & 27.27 \\
\hline 129 & Sludge + MST & 35 & 4.2 & 17.53 & 20.36 \\
\hline 130 & Sludge + MST & 35 & 4.2 & 20.59 & 27.53 \\
\hline 131 & Sludge + MST & 35 & 4.2 & 23.58 & 35.53 \\
\hline 132 & Sludge + MST & 35 & 4.2 & 26.44 & 44.25 \\
\hline 133 & Sludge + MST & 35 & 4.2 & 21.64 & 30.58 \\
\hline 134 & Sludge + MST & 35 & 4.2 & 16.76 & 19.13 \\
\hline 135 & Sludge + MST & 35 & 4.2 & 11.87 & 10.06 \\
\hline 136 & Sludge + MST & 35 & 4.2 & 16.75 & 19.16 \\
\hline
\end{tabular}




\begin{tabular}{|c|c|c|c|c|c|}
\hline Test & Feed & Temp & Conc (wt \%) & $\underline{\text { Vel }(\mathrm{ft} / \mathrm{s})}$ & $\underline{\mathrm{DP}}(\mathrm{psi})$ \\
\hline$\overline{137}$ & Sludge + MST & 35 & 4.2 & 21.58 & 30.56 \\
\hline 138 & Sludge+MST & 35 & 4.2 & 26.44 & 44.19 \\
\hline 139 & Sludge +MST & 35 & 4.2 & 20.79 & 28.32 \\
\hline 140 & Sludge+MST & 35 & 4.2 & 17.88 & 21.34 \\
\hline 141 & Sludge $+\mathrm{MST}$ & 35 & 4.2 & 11.84 & 9.72 \\
\hline 142 & Sludge + MST & 35 & 4.2 & 13.94 & 13.40 \\
\hline 143 & Sludge+MST & 35 & 4.2 & 16.80 & 18.89 \\
\hline 144 & Sludge+MST & 35 & 4.2 & 11.87 & 10.05 \\
\hline 145 & Sludge+MST & 35 & 4.2 & 11.87 & 10.08 \\
\hline 146 & Sludge+MST & 35 & 4.2 & 11.83 & 9.95 \\
\hline 147 & Sludge + MST & 35 & 4.2 & 11.83 & 9.99 \\
\hline 148 & Sludge+MST & 35 & 4.2 & 11.87 & 10.14 \\
\hline 149 & Sludge + MST & 35 & 4.2 & 16.85 & 19.22 \\
\hline 150 & Sludge+MST & 35 & 4.2 & 25.93 & 42.94 \\
\hline 151 & Sludge + MST & 35 & 4.2 & 16.85 & 19.25 \\
\hline 152 & Sludge + MST & 35 & 4.2 & 15.78 & 17.11 \\
\hline 153 & Sludge + MST & 35 & 4.2 & 15.84 & 17.24 \\
\hline 154 & Sludge + MST & 35 & 4.2 & 11.84 & 9.82 \\
\hline 37 & 6.4 M Sludge+MST & 35 & 0.05 & 8.97 & 6.69 \\
\hline 38 & 6.4 M Sludge+MST & 35 & 0.05 & 11.94 & 11.33 \\
\hline 39 & 6.4 M Sludge+MST & 35 & 0.05 & 4.04 & 1.54 \\
\hline 40 & 6.4 M Sludge+MST & 35 & 0.05 & 8.93 & 6.59 \\
\hline 41 & 6.4 M Sludge+MST & 35 & 0.05 & 11.90 & 11.15 \\
\hline 42 & 6.4 M Sludge+MST & 35 & 0.05 & 8.96 & 6.61 \\
\hline 43 & 6.4 M Sludge+MST & 35 & 0.05 & 5.98 & 3.16 \\
\hline 44 & 6.4 M Sludge+MST & 35 & 0.05 & 8.98 & 6.64 \\
\hline 45 & 6.4 M Sludge+MST & 35 & 0.05 & 14.01 & 14.92 \\
\hline 46 & 6.4 M Sludge+MST & 35 & 0.05 & 5.96 & 2.97 \\
\hline 47 & 6.4 M Sludge+MST & 35 & 0.05 & 8.95 & 6.49 \\
\hline 52 & 6.4 M Sludge+MST & 35 & 0.05 & 8.94 & 6.58 \\
\hline 53 & 6.4 M Sludge+MST & 35 & 0.05 & 11.92 & 11.16 \\
\hline 54 & 6.4 M Sludge+MST & 35 & 0.05 & 4.02 & 1.47 \\
\hline 55 & 6.4 M Sludge+MST & 35 & 0.05 & 8.91 & 6.51 \\
\hline 56 & 6.4 M Sludge+MST & 35 & 0.05 & 11.89 & 11.02 \\
\hline 57 & 6.4 M Sludge+MST & 35 & 0.05 & 8.94 & 6.50 \\
\hline 58 & 6.4 M Sludge+MST & 35 & 0.05 & 5.97 & 3.06 \\
\hline 59 & 6.4 M Sludge+MST & 35 & 0.05 & 8.95 & 6.54 \\
\hline 60 & 6.4 M Sludge+MST & 35 & 0.05 & 14.00 & 14.78 \\
\hline 61 & 6.4 M Sludge+MST & 35 & 0.05 & 5.93 & 2.92 \\
\hline 62 & 6.4 M Sludge+MST & 35 & 0.05 & 8.93 & 6.44 \\
\hline 66 & 6.4 M Sludge+MST & 35 & 0.74 & 8.93 & 6.71 \\
\hline 67 & 6.4 M Sludge+MST & 35 & 0.74 & 11.90 & 11.22 \\
\hline 68 & 6.4 M Sludge+MST & 35 & 0.74 & 3.99 & 1.61 \\
\hline 69 & 6.4 M Sludge+MST & 35 & 0.74 & 8.86 & 6.42 \\
\hline 70 & 6.4 M Sludge+MST & 35 & 0.74 & 11.88 & 11.03 \\
\hline 71 & 6.4 M Sludge+MST & 35 & 0.74 & 8.93 & 5.58 \\
\hline 72 & 6.4 M Sludge+MST & 35 & 0.74 & 5.93 & 3.31 \\
\hline 73 & 6.4 M Sludge+MST & 35 & 0.74 & 8.92 & 6.89 \\
\hline 74 & 6.4 M Sludge+MST & 35 & 0.74 & 13.98 & 14.90 \\
\hline 75 & 6.4 M Sludge+MST & 35 & 0.74 & 5.92 & 3.06 \\
\hline
\end{tabular}




\begin{tabular}{|c|c|c|c|c|c|}
\hline Test & Feed & $\underline{\text { Temp }}$ & Conc (wt \%) & $\underline{\mathrm{Vel}}(\mathrm{ft} / \mathrm{s})$ & $\underline{\mathrm{DP}}(\mathrm{psi})$ \\
\hline$\overline{76}$ & 6.4 M Sludge + MST & 35 & 0.74 & 8.92 & 6.66 \\
\hline 81 & 6.4 M Sludge+MST & 35 & 0.74 & 8.92 & 6.70 \\
\hline 82 & 6.4 M Sludge+MST & 35 & 0.74 & 11.90 & 11.29 \\
\hline 83 & 6.4 M Sludge+MST & 35 & 0.74 & 3.98 & 1.66 \\
\hline 84 & 6.4 M Sludge+MST & 35 & 0.74 & 8.90 & 6.59 \\
\hline 85 & 6.4 M Sludge+MST & 35 & 0.74 & 11.88 & 11.07 \\
\hline 86 & 6.4 M Sludge+MST & 35 & 0.74 & 8.92 & 6.63 \\
\hline 87 & 6.4 M Sludge+MST & 35 & 0.74 & 5.93 & 3.32 \\
\hline 88 & 6.4 M Sludge+MST & 35 & 0.74 & 8.92 & 6.76 \\
\hline 89 & 6.4 M Sludge+MST & 35 & 0.74 & 13.97 & 14.83 \\
\hline 90 & 6.4 M Sludge+MST & 35 & 0.74 & 5.91 & 2.97 \\
\hline 91 & 6.4 M Sludge+MST & 35 & 0.74 & 8.91 & 6.53 \\
\hline 95 & 6.4 M Sludge+MST & 35 & 0.05 & 8.93 & 6.13 \\
\hline 96 & 6.4 M Sludge+MST & 35 & 0.05 & 11.94 & 10.79 \\
\hline 97 & 6.4 M Sludge+MST & 35 & 0.05 & 4.01 & 1.25 \\
\hline 98 & 6.4 M Sludge+MST & 35 & 0.05 & 8.90 & 6.00 \\
\hline 99 & 6.4 M Sludge+MST & 35 & 0.05 & 11.87 & 10.44 \\
\hline 100 & 6.4 M Sludge+MST & 35 & 0.05 & 8.93 & 6.21 \\
\hline 101 & 6.4 M Sludge+MST & 35 & 0.05 & 5.96 & 2.87 \\
\hline 102 & 6.4 M Sludge+MST & 35 & 0.05 & 8.94 & 6.27 \\
\hline 103 & 6.4 M Sludge+MST & 35 & 0.05 & 13.97 & 14.24 \\
\hline 104 & 6.4 M Sludge+MST & 35 & 0.05 & 5.93 & 2.69 \\
\hline 105 & 6.4 M Sludge+MST & 35 & 0.05 & 8.93 & 6.22 \\
\hline 110 & 6.4 M Sludge+MST & 35 & 0.05 & 8.93 & 6.34 \\
\hline 111 & 6.4 M Sludge+MST & 35 & 0.05 & 11.89 & 10.90 \\
\hline 112 & 6.4 M Sludge+MST & 35 & 0.05 & 4.02 & 1.26 \\
\hline 113 & 6.4 M Sludge+MST & 35 & 0.05 & 8.90 & 6.25 \\
\hline 114 & 6.4 M Sludge+MST & 35 & 0.05 & 11.87 & 10.74 \\
\hline 115 & 6.4 M Sludge+MST & 35 & 0.05 & 8.92 & 6.28 \\
\hline 116 & 6.4 M Sludge+MST & 35 & 0.05 & 5.95 & 2.88 \\
\hline 117 & 6.4 M Sludge+MST & 35 & 0.05 & 8.94 & 6.31 \\
\hline 118 & 6.4 M Sludge+MST & 35 & 0.05 & 13.97 & 14.52 \\
\hline 119 & 6.4 M Sludge+MST & 35 & 0.05 & 5.92 & 2.78 \\
\hline 120 & 6.4 M Sludge+MST & 35 & 0.05 & 8.92 & 6.27 \\
\hline 124 & 6.4 M Sludge+MST & 35 & 0.1 & 8.92 & 6.40 \\
\hline 125 & 6.4 M Sludge+MST & 35 & 0.1 & 11.90 & 10.93 \\
\hline 126 & 6.4 M Sludge+MST & 35 & 0.1 & 4.01 & 1.32 \\
\hline 127 & 6.4 M Sludge+MST & 35 & 0.1 & 8.90 & 6.32 \\
\hline 128 & 6.4 M Sludge+MST & 35 & 0.1 & 11.87 & 10.85 \\
\hline 129 & 6.4 M Sludge+MST & 35 & 0.1 & 8.92 & 6.36 \\
\hline 130 & 6.4 M Sludge+MST & 35 & 0.1 & 5.95 & 2.97 \\
\hline 131 & 6.4 M Sludge+MST & 35 & 0.1 & 8.94 & 6.40 \\
\hline 132 & 6.4 M Sludge+MST & 35 & 0.1 & 13.97 & 14.60 \\
\hline 133 & 6.4 M Sludge+MST & 35 & 0.1 & 5.92 & 2.86 \\
\hline 134 & 6.4 M Sludge+MST & 35 & 0.1 & 8.92 & 6.34 \\
\hline 139 & 6.4 M Sludge+MST & 35 & 0.1 & 8.93 & 6.46 \\
\hline 140 & 6.4 M Sludge+MST & 35 & 0.1 & 11.89 & 11.00 \\
\hline 141 & 6.4 M Sludge+MST & 35 & 0.1 & 4.00 & 1.38 \\
\hline 142 & 6.4 M Sludge+MST & 35 & 0.1 & 8.90 & 6.27 \\
\hline 143 & 6.4 M Sludge+MST & 35 & 0.1 & 11.86 & 10.78 \\
\hline 144 & 6.4 M Sludge+MST & 35 & 0.1 & 8.92 & 6.33 \\
\hline
\end{tabular}




\begin{tabular}{|c|c|c|c|c|c|}
\hline Test & Feed & $\underline{\text { Temp }}$ & Conc (wt \%) & $\underline{\mathrm{Vel}}(\mathrm{ft} / \mathrm{s})$ & $\underline{\mathrm{DP}}$ (psi) \\
\hline 145 & 6.4 M Sludge+MST & 35 & 0.1 & 5.94 & 2.95 \\
\hline 146 & 6.4 M Sludge+MST & 35 & 0.1 & 8.94 & 6.37 \\
\hline 147 & 6.4 M Sludge+MST & 35 & 0.1 & 13.96 & 14.55 \\
\hline 148 & 6.4 M Sludge+MST & 35 & 0.1 & 5.92 & 2.82 \\
\hline 149 & 6.4 M Sludge+MST & 35 & 0.1 & 8.92 & 6.31 \\
\hline 153 & 6.4 M Sludge+MST & 35 & 0.2 & 8.92 & 6.50 \\
\hline 154 & 6.4 M Sludge+MST & 35 & 0.2 & 11.89 & 11.07 \\
\hline 155 & 6.4 M Sludge+MST & 35 & 0.2 & 4.00 & 1.45 \\
\hline 156 & 6.4 M Sludge+MST & 35 & 0.2 & 8.90 & 6.31 \\
\hline 157 & 6.4 M Sludge+MST & 35 & 0.2 & 11.86 & 10.84 \\
\hline 158 & 6.4 M Sludge+MST & 35 & 0.2 & 8.91 & 6.38 \\
\hline 159 & 6.4 M Sludge+MST & 35 & 0.2 & 5.94 & 2.98 \\
\hline 160 & 6.4 M Sludge+MST & 35 & 0.2 & 8.93 & 6.41 \\
\hline 161 & 6.4 M Sludge+MST & 35 & 0.2 & 13.95 & 14.67 \\
\hline 162 & 6.4 M Sludge+MST & 35 & 0.2 & 5.92 & 2.89 \\
\hline 163 & 6.4 M Sludge+MST & 35 & 0.2 & 8.92 & 6.35 \\
\hline 168 & 6.4 M Sludge+MST & 35 & 0.2 & 8.91 & 6.48 \\
\hline 169 & 6.4 M Sludge+MST & 35 & 0.2 & 11.85 & 10.99 \\
\hline 170 & 6.4 M Sludge+MST & 35 & 0.2 & 3.99 & 1.38 \\
\hline 171 & 6.4 M Sludge+MST & 35 & 0.2 & 8.88 & 6.34 \\
\hline 172 & 6.4 M Sludge+MST & 35 & 0.2 & 11.87 & 10.89 \\
\hline 173 & 6.4 M Sludge+MST & 35 & 0.2 & 8.91 & 6.38 \\
\hline 174 & 6.4 M Sludge+MST & 35 & 0.2 & 5.95 & 3.00 \\
\hline 175 & 6.4 M Sludge+MST & 35 & 0.2 & 8.93 & 6.42 \\
\hline 176 & 6.4 M Sludge+MST & 35 & 0.2 & 13.97 & 14.69 \\
\hline 177 & 6.4 M Sludge+MST & 35 & 0.2 & 5.91 & 2.87 \\
\hline 178 & 6.4 M Sludge+MST & 35 & 0.2 & 8.91 & 6.36 \\
\hline 182 & 6.4 M Sludge+MST & 35 & 0.39 & 8.90 & 6.62 \\
\hline 183 & 6.4 M Sludge+MST & 35 & 0.39 & 11.88 & 11.21 \\
\hline 184 & 6.4 M Sludge+MST & 35 & 0.39 & 3.99 & 1.58 \\
\hline 185 & 6.4 M Sludge+MST & 35 & 0.39 & 8.89 & 6.48 \\
\hline 186 & 6.4 M Sludge+MST & 35 & 0.39 & 11.86 & 11.05 \\
\hline 187 & 6.4 M Sludge+MST & 35 & 0.39 & 8.90 & 6.54 \\
\hline 188 & 6.4 M Sludge+MST & 35 & 0.39 & 5.92 & 3.18 \\
\hline 189 & 6.4 M Sludge+MST & 35 & 0.39 & 8.92 & 6.62 \\
\hline 190 & 6.4 M Sludge+MST & 35 & 0.39 & 13.97 & 14.94 \\
\hline 191 & 6.4 M Sludge+MST & 35 & 0.39 & 5.93 & 3.07 \\
\hline 192 & 6.4 M Sludge+MST & 35 & 0.39 & 8.88 & 6.51 \\
\hline 197 & 6.4 M Sludge+MST & 35 & 0.39 & 8.90 & 6.75 \\
\hline 198 & 6.4 M Sludge+MST & 35 & 0.39 & 11.87 & 11.33 \\
\hline 199 & 6.4 M Sludge+MST & 35 & 0.39 & 3.98 & 1.61 \\
\hline 200 & 6.4 M Sludge+MST & 35 & 0.39 & 8.92 & 6.55 \\
\hline 201 & 6.4 M Sludge+MST & 35 & 0.39 & 11.85 & 11.07 \\
\hline 202 & 6.4 M Sludge+MST & 35 & 0.39 & 8.90 & 6.53 \\
\hline 203 & 6.4 M Sludge+MST & 35 & 0.39 & 5.92 & 3.14 \\
\hline 204 & 6.4 M Sludge+MST & 35 & 0.39 & 8.91 & 6.55 \\
\hline 205 & 6.4 M Sludge+MST & 35 & 0.39 & 13.95 & 14.81 \\
\hline 206 & 6.4 M Sludge+MST & 35 & 0.39 & 5.91 & 2.99 \\
\hline 207 & 6.4 M Sludge+MST & 35 & 0.39 & 8.90 & 6.49 \\
\hline
\end{tabular}

